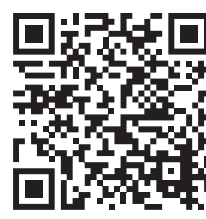

\title{
Coriorretinitis con afectación macular como complicación en enfermedad de Kikuchi-Fujimoto con sobreposición de lupus eritematoso sistémico
}

\author{
José Guillermo Murguía Pérez, ${ }^{\star}$ Rosalía Heras Salazar,‡ Miguel García Domínguez§
}

\section{RESUMEN}

Introducción: La enfermedad de Kikuchi-Fujimoto (EKF), también conocida como linfadenitis necrosante histiocítica de Kikuchi, es una condición rara y benigna de causa desconocida. En la literatura hay reportes de casos de lupus eritematoso sistémico (LES) en coexistencia con EKF, siendo motivo de controversia si representa una manifestación clínica propia de LES o un proceso concomitante. Si bien es poco frecuente esta asociación, pueden existir manifestaciones poco usuales. Reporte de caso: Femenino de 16 años de edad con diagnóstico de EKF con sobreposición con LES, quien desarrolló como complicación aguda coriorretinitis con afectación macular de ojo izquierdo. Ameritó tratamiento con bolos de metilprednisolona, inmunosupresor y aplicación intravítrea de esteroide, actualmente con remisión de sintomatología sistémica y disminución importante de agudeza visual de ojo izquierdo. Conclusión: Las participación ocular debe ser considerada en la evaluación inicial de enfermedades sistémicas, autoinmunes o con sobreposición, para evitar complicaciones que puedan ser irreversibles.

Palabras clave: Kikuchi-Fujimoto, lupus eritematoso sistémico, linfadenopatía, coriorretinitis, uveítis posterior.

\section{ABSTRACT}

Introduction: Kikuchi-Fujimoto's disease (KFD), also known as Kikuchi histiocytic necrotizing lymphadenitis, is a rare and benign condition of unknown cause. In the literature, there are reports of cases of systemic lupus erythematosus (SLE) in coexistence with EKF, and it is controversial whether it represents a clinical manifestation of SLE or a concomitant process. Although this association is rare, there may be unusual manifestations. Case report: A 16-year-old female with a diagnosis of EKF overlap with SLE who developed as an acute complication chorioretinitis with macular involvement of the left eye. She was treated with methylprednisolone boluses, immunosuppressant and intravitreal steroid application, currently with remission of systemic symptoms and an important decrease in visual acuity of the left eye. Conclusion: Eye involvement should be considered in the initial evaluation of systemic, autoimmune or overlapping diseases to avoid complications that may be irreversible.

Keywords: Kikuchi-Fujimoto, systemic lupus erythematosus, lymphadenopathy, chorioretinitis, posterior uveitis.

\footnotetext{
* Departamento de Pediatría Médica.

‡ Departamento de Oftalmología Pediátrica.

$\S$ Departamento de Inmunología y Alergia.
} 
Vol. 29, Núm. 2 • Mayo-Agosto 2020

\section{INTRODUCCIÓN}

La enfermedad de Kikuchi-Fujimoto (EKF), también conocida como linfadenitis necrosante histiocítica de Kikuchi, es una condición rara y benigna de causa desconocida, caracterizada por linfadenitis necrosante subaguda cervical, que muestra hiperplasia focal de células reticulares con restos nucleares y fagocitosis, acompañado de fiebre y leucopenia, usualmente en mujeres asiáticas de la tercera década de la vida previamente sanas, aunque puede afectar a todas las razas. ${ }^{1,2}$ Se ha relacionado con mecanismos infecciosos y/o autoinmunes, como enfermedad mixta del tejido conectivo, enfermedad de Still para lupus eritematoso sistémico (LES) en 13 a $25 \%, 3,4$ que pueden manifestarse antes o después al diagnóstico de EKF; además de las manifestaciones clásicas, se tiene el reporte de alteraciones en diferentes aparatos y sistemas, poco habituales a nivel ocular para EKF, mientras que en LES la afección varía de $0.1 \%$ a $4.8 \% .^{5}$ La EKF se describió inicialmente en mujeres jóvenes, aunque ocurre también en varones, con una relación de 1:4 a 1:6, mientras que en México se desconoce la prevalencia de esta enfermedad. ${ }^{6}$ La histopatología de los ganglios linfáticos afectados es la clave diagnóstica, en donde se distinguen tres fases: proliferativa, necrosante y xantomatosa.

\section{CASO CLÍNICO}

Femenino de 16 años de edad, sin antecedentes heredofamiliares. Inició dos meses previos con fiebre intermitente, astenia, adinamia, cefalea holocraneal, cervicalgia con edema de tejidos blandos no delimitado. Al
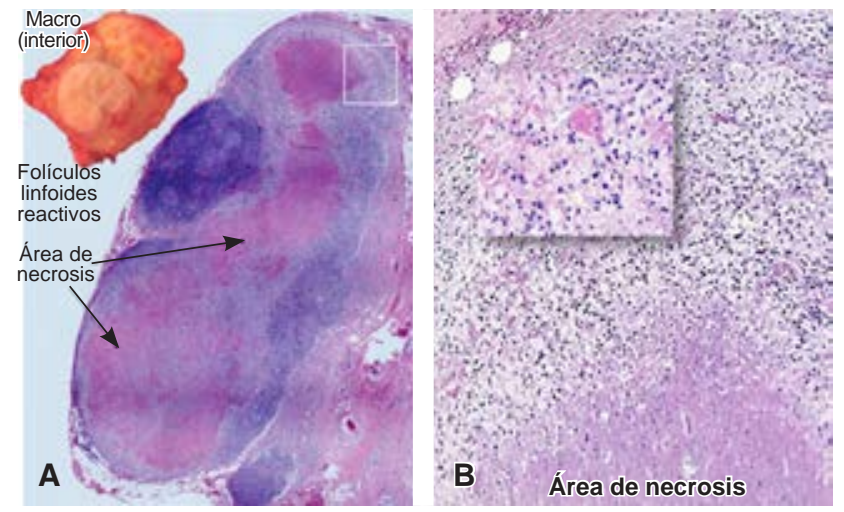

Figura 1: A) Biopsia de ganglio linfático: hiperplasia folicular mixta (paracortical y folicular), con áreas de necrosis geográfica. B) Periferia con macrófagos y detritus nucleares.
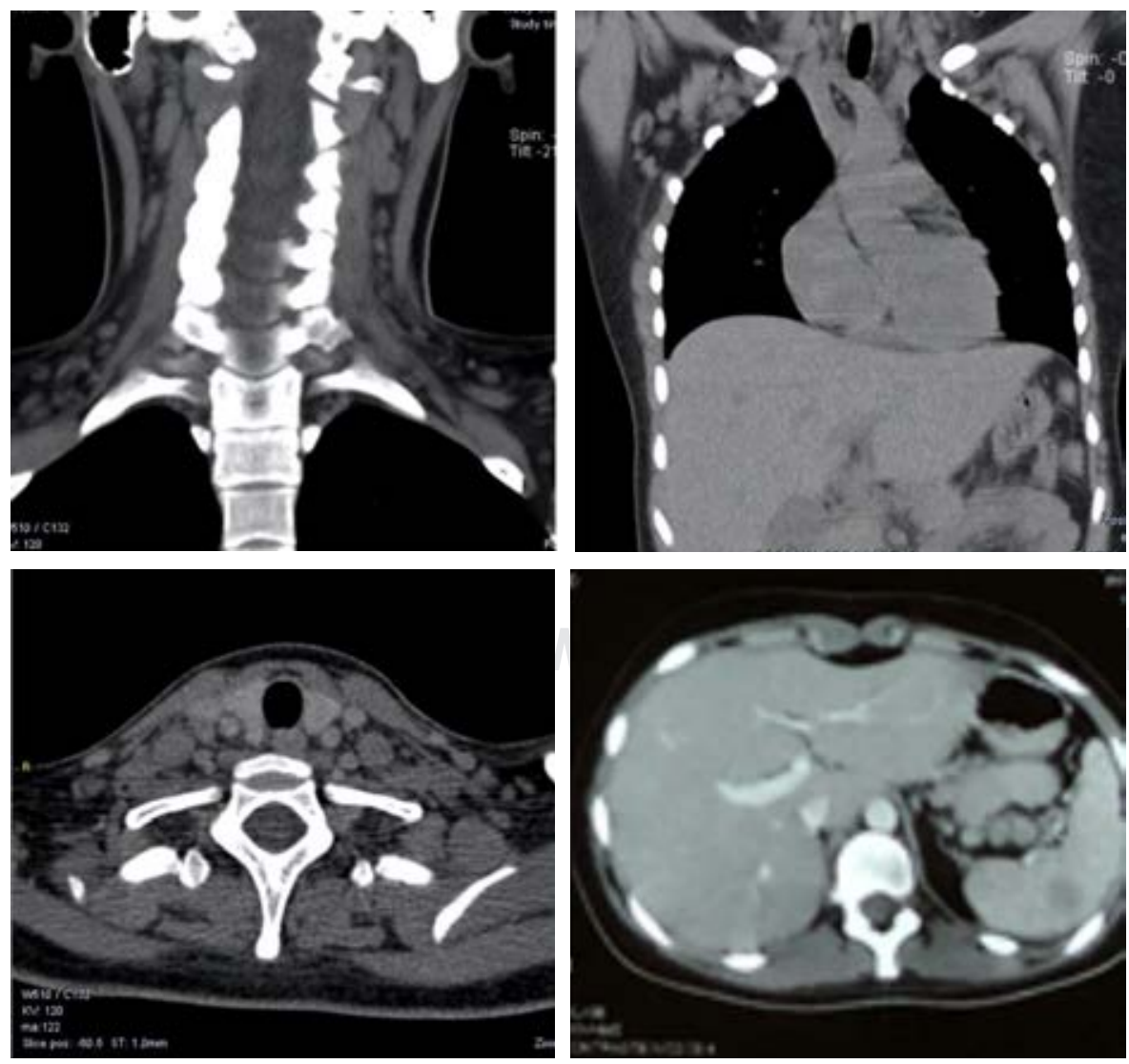

Figura 2:

Adenopatías cervicales, axilares y mediastinales con eje corto no mayor a $66 \mathrm{~mm}$. En la fase contrastada se observa una lesión redondeada en bazo con densidad de $120 \mathrm{uH}$ (en fase simple $40 \mathrm{uH}$ ) de $26 \times 18 \mathrm{~mm}$, múltiples adenopatías ovaladas sobre su hilio de $13 \mathrm{~mm}$. Conglomerados ganglionares retroperitoneales bilaterales, el mayor de $56 \times 16 \mathrm{~mm}$. 
Vol. 29, Núm. 2 • Mayo-Agosto 2020

mes presenta adenomegalias cervicales, artralgias en columna vertebral, náusea, vómito y pérdida ponderal de 5 kilos. La tomografía (TC) toracoabdominal demostró conglomerado ganglionar cervical, parahiliar pulmonar y retroperitoneal abdominal, se realizó biopsia de ganglio cervical que reportó hiperplasia folicular reactiva con áreas de necrosis geográfica, identificando macrófagos con detritus nucleares en la periferia sugerente de EKF (Figura 1), motivo de referencia a nuestro hospital.

En la exploración física se observó caída de cabello, artralgias en ambas muñecas, cervicales y dorsolumbares, palidez generalizada y adenomegalias cervicales blandas, móviles, no dolorosas, no adheridas a planos profundos, de aproximadamente $6 \times 10 \mathrm{~mm}$, sin hepatoni esplenomegalia. La TC contrastada cérvico-toracoabdominal reportó hallazgos compatibles con proceso ganglionar neoproliferativo en región cervical y axilar bilateral, parahiliar pulmonar, retroperitoneal y en bazo (Figura 2). El reporte de inmunohistoquímica fue negativo a neoplasia (CD20 positivo en linfocitos $B$ reactivos, CD5 positivo en linfocitos $T$ reactivos, mieloperoxidasa positivo en macrófagos) con CD123 positivo multifocal, en aéreas perilesionales.

Las pruebas complementarias reportaron linfopenia, anticuerpos antinucleares (ANA) positivos $(1: 1,280)$, antiDNA nativo positivo (1:320), anticuerpos anti-Smith positivo, hipocomplementemia, Coombs directo positivo y perfil de antifosfolípidos positivo, integrando el diagnóstico de LES con 8/17 criterios de SLICC 2017 (Tabla 1).

A las 72 horas de su ingreso, presentó disminución de agudeza visual bilateral, ojo derecho (OD) 10/10 cerca, 20/20 lejos, fondo de ojo (FO) con tortuosidad vas-

Tabla 1: Criterios diagnósticos de lupus eritematoso sistémico (LES) y enfermedad de Kikuchi-Fujimoto (EKF). Características clínicas y de laboratorio del paciente (en cursiva).

\begin{tabular}{|c|c|c|}
\hline \multirow[t]{7}{*}{ Clínicas } & SLICC 2017 & EKF \\
\hline & Lupus cutáneo agudo. Incluye eritema malar, fotosensibilidad & Linfadenopatías \\
\hline & Lupus cutáneo crónico. Incluye lupus discoide & Rash \\
\hline & Alopecia no cicatricial & Fiebre \\
\hline & Úlceras orales/nasales & Artritis \\
\hline & Sinovitis. Incluye artritis & Hepatoesplenomegalia \\
\hline & Serositis. Incluye pleuritis/pericarditis & Fatiga \\
\hline \multirow[t]{17}{*}{ Laboratorio } & Renal & Reporte histopatológico \\
\hline & Proteinuria $>0.5 \mathrm{~g} / \mathrm{dí} a$ & \\
\hline & Cilindros hemáticos & \\
\hline & Neurológico & Leucopenia \\
\hline & Incluye crisis convulsivas y psicosis & \\
\hline & Anemia hemolítica & Inmunohistoquímica positiva CD68 \\
\hline & Coombs directo positivo en ausencia de anemia hemolítica & \\
\hline & Leucopenia $<4.000 / \mathrm{mm}^{3}$ (linfopenia $<1.000 / \mathrm{mm}^{3}$ ) & \\
\hline & Trombocitopenia $\left(<100.000 / \mathrm{mm}^{3}\right)$ & \\
\hline & ANA & \\
\hline & Anti-ADN de doble cadena & \\
\hline & Anti-Smith & \\
\hline & Anti-fosfolípidos & \\
\hline & Anticoagulante lúpico/reagina plasmática Anticardiolipinas & \\
\hline & Anti- $\beta 2 g p$ I & \\
\hline & Complemento bajo & \\
\hline & $\mathrm{C} 3, \mathrm{C} 4$ o $\mathrm{CH} 50$ & \\
\hline
\end{tabular}

Hemoglobina $9.4 \mathrm{~g} / \mathrm{dL}$, Hto. $29 \%$, leucocitos $5.900 / \mathrm{mm}^{3}$, neutrófilos $4.840 / \mathrm{mm}^{3}$, linfocitos $944 / \mathrm{mm}^{3}$, plaquetas $371.000 / \mathrm{mm}^{3}$, velocidad de sedimentación globular $47 \mathrm{~mm} / \mathrm{h}$, proteína C reactiva $3.75 \mathrm{mg} / \mathrm{dL}$, DHL $566 \mathrm{U} / \mathrm{L}$, bilirrubina total $0.5 \mathrm{mg} / \mathrm{dL}$, bilirrubina directa $0.1 \mathrm{mg} / \mathrm{dL}$, AST $116 \mathrm{U} / \mathrm{L}$, ALT $106 \mathrm{U} / \mathrm{L}$, albúmina $3.3 \mathrm{mg} / \mathrm{dL}$, ferritina $2.251 \mathrm{mg} / \mathrm{dL}$. Negativo a TORCH, VEB, BAAR, tinción Z-N, VIH, parasitosis, Brucella, VHS 1 y 2.

$\operatorname{lgG} 1.841 \mathrm{mg} / \mathrm{dL}, \lg A 377 \mathrm{mg} / \mathrm{dL}$, IgM $51 \mathrm{mg} / \mathrm{dL}, \operatorname{lgE} 50 \mathrm{U} / \mathrm{L}$.

ANA 1:1,280 moteado fino, anti-DNA nativo 1:320, anti-Smith 30.9 (<20), anti-Ro 6.1, anti-La 1.7, Coombs directo 1:2, anti beta 2 glucoproteína IgM en 24 ( $<20)$, antibeta 2 glucoproteína lgG en 2, anticardiolipinas IgG 5.7 e lgM 4.5. Anticoagulante lúpico positivo. C3 de $80 \mathrm{mg} / \mathrm{dL}, \mathrm{C} 416.4 \mathrm{mg} / \mathrm{dL}$. Proteínas en orina de $24 \mathrm{~h} 0.25 \mathrm{~g} / \mathrm{d}$. Factor reumatoide negativo.

SLICC = Systemic Lupus International Collaborating Clinics; ANA = anticuerpos antinucleares; Hto. = hematocrito; DHL = deshidrogenasa láctica; AST = aspartato aminotransferasa; $\mathrm{ALT}$ = alanina aminotransferasa; $\mathrm{TORCH}$ = toxoplasmosis, rubéola, citomegalovirus, herpes simple y VIH [por sus iniciales en inglés]; VEB = virus de Epstein-Barr; BAAR = bacilos ácido-alcohol resistentes; $\mathrm{VIH}$ = virus de la inmunodeficiencia humana; VHS = virus del herpes simple; IgG = inmunoglobulina G; IgA = inmunoglobulina $\mathrm{A}$; IgM = inmunoglobulina $\mathrm{M}$; IgE = inmunoglobulina $\mathrm{E}$. 
Vol. 29, Núm. 2 • Mayo-Agosto 2020
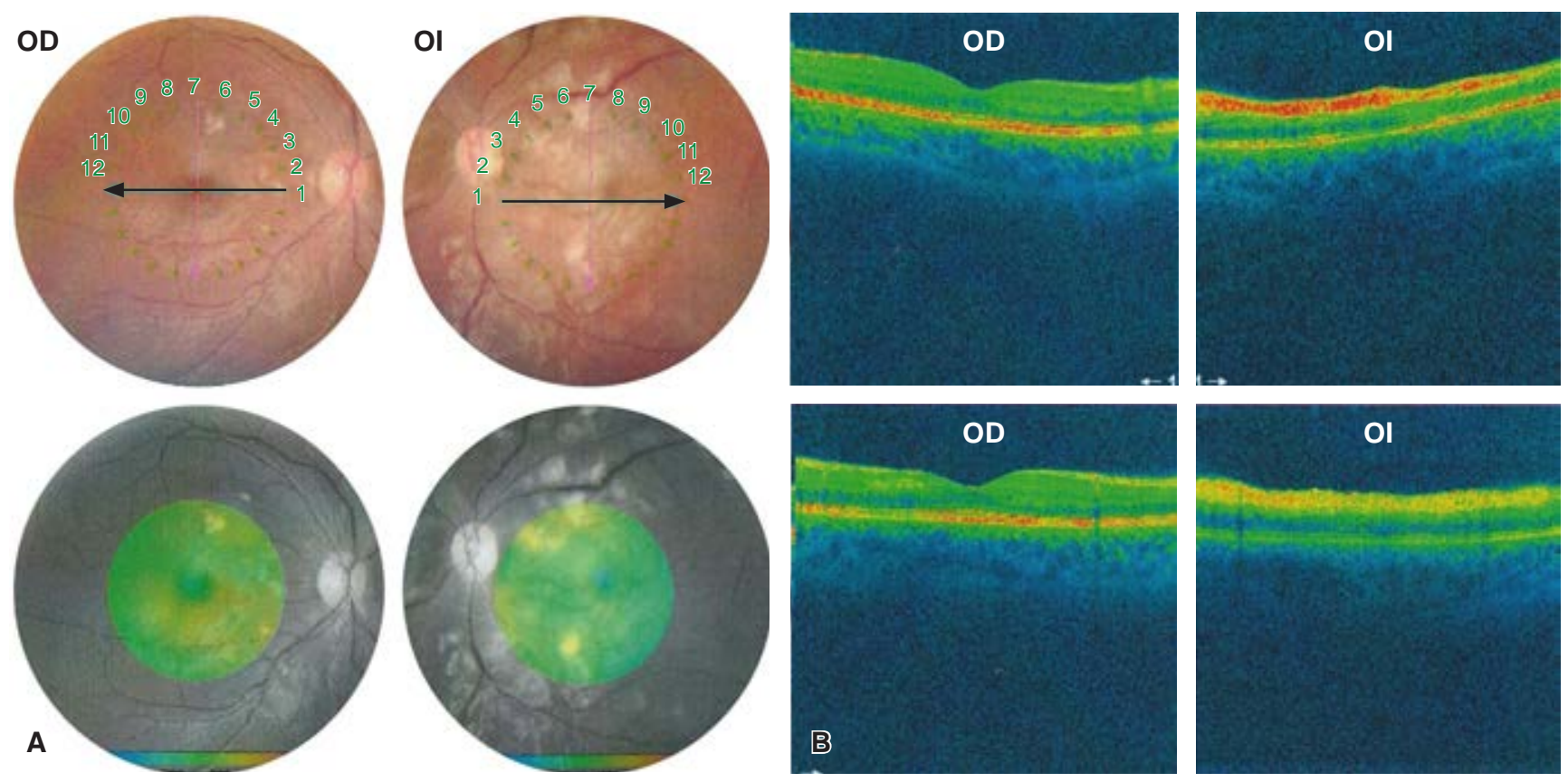

Figura 3: A) Fondo de ojo derecho (OD) e izquierdo (OI): vasos de emergencia central tortuosos, infiltrados coriorretinianos algodonosos con levantamiento seroso en el sector temporal superior con compromiso foveal de predominio en OI. B) Tomografía óptica coherente. OD: área macular con grosor central de $240 \mu \mathrm{m}$, adecuada depresión foveal; corte central: área de sector superior con disminución del grosor macular. OI: área macular con grosor central de 261 m, depresión foveal ausente; corte central: disminución del grosor macular combinado con áreas de atrofia y edema; capa de fibras nerviosas: áreas con grosor normal combinada con áreas de edema del nervio óptico.

cular; ojo izquierdo (OI): agudeza visual a cuenta-dedos $50 \mathrm{~cm}$, FO con vasos de emergencia central tortuosos, polo posterior con infiltrados coriorretinianos algodonosos, levantamiento seroso en sector temporal superior con compromiso de fóvea (Figura 3).

Se realizó tomografía óptica coherente (OCT), la cual reportó en OD: área macular con grosor central de 240 micras, buena depresión foveal, sin alteraciones de la interface vitreorretiniana, corte más central de un área disminuida en el grosor macular en sector superior. En OI: área macular con grosor central de 261 micras, depresión foveal ausente, corte central, se observan áreas con disminución del grosor macular, sector superior con aumento del grosor, combinando áreas de atrofia y edema, capa de fibras nerviosas mantiene áreas de grosor normal y áreas con edema del nervio óptico (Figura 3).

Debido al compromiso súbito de la agudeza visual y hallazgos del FO, se inició tratamiento con bolos de metilprednisolona a $30 \mathrm{mg} / \mathrm{kg} /$ día por tres días, micofenolato de mofetilo a $2 \mathrm{~g} /$ día durante tres días. Se observó mejoría de síntomas constitucionales y reducción de adenomegalias. La revaloración oftalmológica determinó discreta mejoría de agudeza visual con persistencia de inflamación de predominio en OI, por lo que se decidió la aplicación intravítrea de es- teroide de depósito ocular a $0.7 \mathrm{mg}$ en ambos ojos como dosis única.

Se egresó con micofenolato de mofetilo a 2 g/día, hidroxicloroquina a $3 \mathrm{mg} / \mathrm{kg} /$ día y prednisona a $1 \mathrm{mg} /$ $\mathrm{kg} /$ día. Se mantuvo asintomática con negativización de marcadores de actividad para LES, se realizó a las 12 semanas TC toracoabdominal con ausencia de adenomegalias y el OCT de OD: área macular sin edema, capa de fibras nerviosas normales. OCT de OI: cambios atróficos francos en área macular y marcado deterioro en la capa de fibras nerviosas (Figura 4).

A la exploración de fondo de Ol: nervio óptico con bordes nítidos, palidez ++, red vascular de emergencia central, sin tortuosidad. Engrosamiento de la membrana limitante interna a nivel de nervio óptico y arcadas vasculares, área macular sin brillo foveolar. EI OD: nervio óptico, emergencia de vasos y mácula sin alteraciones.

Actualmente, se encuentra con agudeza visual de OD 20/20, Ol con cuenta-dedos a dos metros, sin actividad sistémica por LES.

\section{DISCUSIÓN}

La asociación de la EKF con el LES es poco frecuente; sin embargo, aún se debate si es coincidencia casual o 
Vol. 29, Núm. $2 \cdot$ Mayo-Agosto 2020

una manifestación clínica de LES. Aunque la mayoría de estudios reportan una evolución benigna, Dorfman ${ }^{7}$ sugiere que EKF puede reflejar una condición autoinmune autolimitada de LES (una forma frustrada). $\mathrm{Hu},{ }^{8}$ basado en la histopatología la EKF concomitante con LES, menciona que se puede tratarse de una linfadenitis lúpica, más que linfadenitis por EKF. Se presenta esta asociación con mayor frecuencia en mujeres (hasta 77\%), edad menor de 30 años (70\%), autolimitada en $64 \%$ de casos, con una tasa de mortalidad de $2.1 \% .{ }^{4} \mathrm{La}$ incidencia de LES-EKF va desde 1.7 a $13.1 \%$, en donde la EKF puede preceder a LES en 1.26 a 2.9\%; presentarse en pacientes con diagnóstico previo de LES en 1.26 a 1.63\%; mientras que la tasa más alta se observa en la presentación simultánea hasta de 2.5 a $9 \%$ (Tabla 2). 3,4,7-29

La afectación de los ganglios linfáticos suele ser cervical y localizada en EKF y, ocasionalmente, el agrandamiento ganglionar puede ser mínimo o sólo mediastínico, incluso ganglios retroperitoneales pueden estar involucrados. ${ }^{12}$ En la mayoría de los estudios comparten algunas características como la elevación de reactantes de fase aguda, VSG incrementada en 70 a $80 \%$ de los casos; proteína C reactiva en 38 a 60\%, DHL en 50 a $80 \%$, leucopenia y ANA en 20 a $40 \%$ de casos adultos y la mitad en edad pediátrica, monocitosis en $26 \%$ y elevación de transaminasas en $25 \%$. 3,6

Las manifestaciones oculares en LES como coroiditis y exudación subretiniana pueden llegar hasta el 3-29\%, a menudo correlacionadas con la actividad de la enfermedad sistémica o preceder otros síntomas sistémi- cos. ${ }^{30,31}$ Mientras que en la EFK son infrecuentes, casos aislados reportan uveítis anterior, panuveítis y edema papilar bilateral, mientras que la vasculitis oclusiva retiniana puede presentarse en ambas entidades. ${ }^{32}$

En ninguno de los estudios mencionados se reporta afección ocular, sólo en casos aislados en forma de isquemia vascular retiniana, retinopatía vaso-oclusiva y oclusión de vena central cuando la actividad de lupus presentó EKF, estableciendo la posibilidad de que el proceso inflamatorio y de procoagulación forman parte de la fisiopatología de esta entidad. ${ }^{33,34}$ En cuanto a reportes de coriorretinitis en EKF-LES no encontramos ninguno.

Nuestra paciente comparte las características descritas con las series publicadas, con la presentación concomitante de LES con vasculitis activa a nivel ocular que condicionó la pérdida súbita de la agudez visual que ameritó de bolos de metilprednisolona, inmunosupresor y esteroide de intravítreo. Además, presentó manifestaciones clínicas concomitantes de LES como de EKF, reconociéndose en tal sentido que estas dos entidades pueden compartir características clínicas y morfológicas, lo cual hace que el diagnóstico diferencial entre ambos sea difícil.

\section{CONCLUSIÓN}

La asociación EFK y LES es poco frecuente y suele ser difícil el diagnóstico al compartir características clínicas y de laboratorio. Sin embargo, la afección ocular puede tener un comportamiento agresivo que comprometa la visión, lo que debe obligar a la realización de evaluacio-
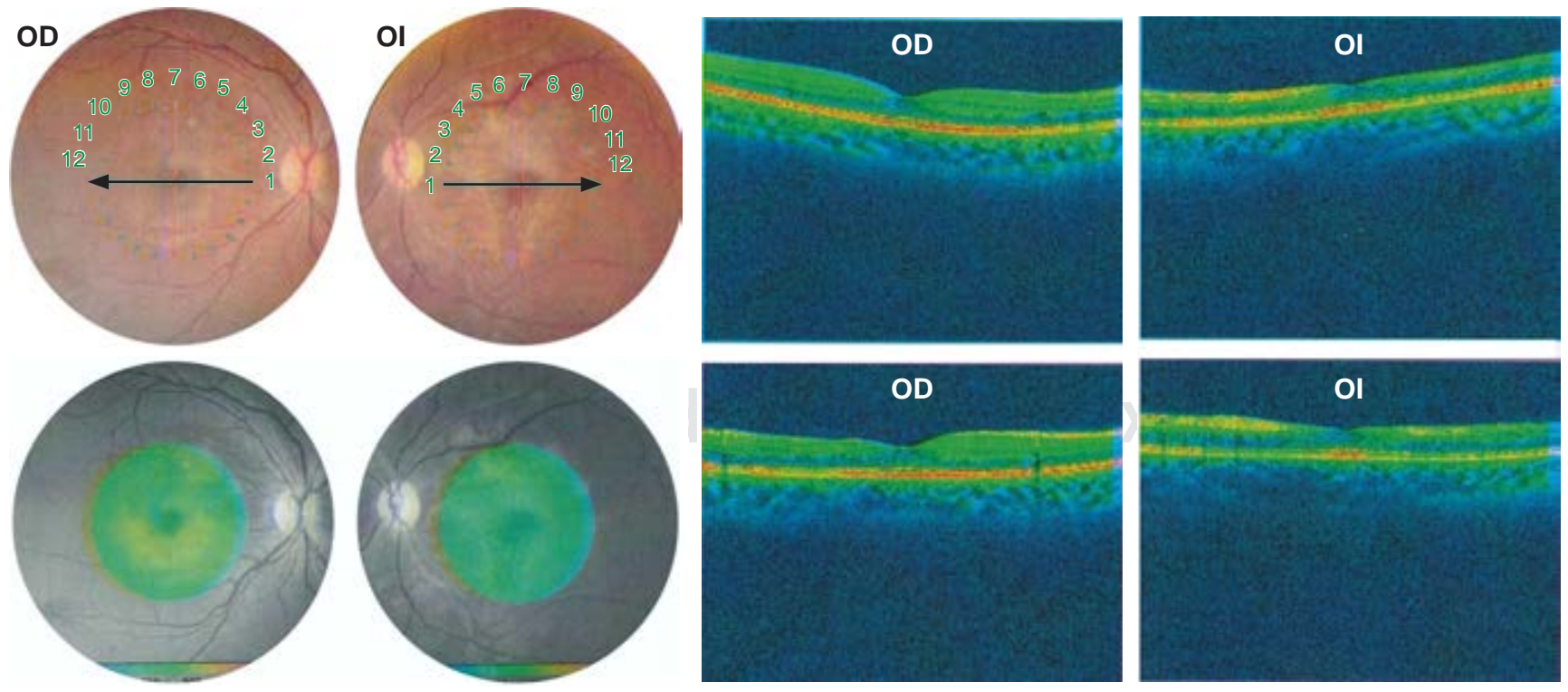

Figura 4: Fondo de ojo y tomografía óptica coherente posterior a aplicación intravítrea de esteroide. Ojo derecho (OD): área macular muestra recuperación de la zona de atrofia, con capa de fibras nerviosas dentro de límites normales. Ojo izquierdo (OI): cambios atróficos francos en área macular y deterioro marcado en la capa de fibras nerviosas. 
Vol. 29, Núm. 2・ Mayo-Agosto 2020

Tabla 2: Estudios reportados de EKF-LES y afección ocular.

\begin{tabular}{|c|c|c|c|c|c|c|}
\hline Autor & No. de casos & $\begin{array}{c}\text { Incidencia } \\
\text { EKF-LES (\%) }\end{array}$ & $\begin{array}{l}\text { EKF antes } \\
\text { de LES (\%) }\end{array}$ & $\begin{array}{c}\text { EKF-LES simultá- } \\
\text { neamente (\%) }\end{array}$ & $\begin{array}{c}\text { EKF después } \\
\text { de LES (\%) }\end{array}$ & $\begin{array}{l}\text { Afección } \\
\text { ocular (\%) }\end{array}$ \\
\hline Pileri et al. ${ }^{9} 1982$ & 27 & 18.50 & 0.00 & 18.50 & 0.00 & 0.00 \\
\hline Turner et al. ${ }^{10} 1983$ & 30 & 0.00 & 0.00 & 0.00 & 0.00 & 0.00 \\
\hline Dorfman et al. ${ }^{7} 1988$ & 108 & 1.85 & 1.85 & 0.00 & 0.00 & 0.00 \\
\hline Cho et al. ${ }^{11} 1991$ & 36 & 5.50 & 5.50 & 0.00 & 0.00 & 0.00 \\
\hline el-Ramahi et al. ${ }^{12} 1994$ & 8 & 25.00 & 25.00 & 0.00 & 0.00 & 0.00 \\
\hline Kuo et al. ${ }^{13} 1995$ & 79 & 5.00 & 1.26 & 2.53 & 1.26 & 0.00 \\
\hline Chen et al. ${ }^{14} 1998$ & 4 & NR & 0.00 & 25.00 & 75.00 & 0.00 \\
\hline Lin et al. ${ }^{15} 2003$ & 61 & 3.30 & 3.30 & 0.00 & 0.00 & 0.00 \\
\hline Hu et al. ${ }^{8} 2003$ & 6 & NR & 16.60 & 66.60 & 16.60 & 0.00 \\
\hline Santana et al. ${ }^{16} 2005$ & 35 & NR & 40.00 & 40.00 & 20.00 & 0.00 \\
\hline Lin et al. ${ }^{17} 2005$ & 23 & 4.34 & 4.34 & 0.00 & 0.00 & 0.00 \\
\hline Chen et al. ${ }^{18} 2005$ & 10 & NR & 16.60 & 100.00 & 16.60 & 0.00 \\
\hline Yu et al. ${ }^{19} 2005$ & 58 & 1.72 & 0.00 & 0.00 & 1.72 & 0.00 \\
\hline Kucukardali et al. ${ }^{4} 2006$ & 244 & 13.10 & 2.45 & $7.3-9^{\star}$ & 1.63 & 0.00 \\
\hline Sanpavat et al. ${ }^{20} 2006$ & 17 & 0.00 & 0.00 & 0.00 & 0.00 & 0.00 \\
\hline Park et al. ${ }^{21} 2007$ & 16 & 0.00 & 0.00 & 0.00 & 0.00 & 0.00 \\
\hline Goldblatt et al. 22008 & 4 & NR & 100.00 & 0.00 & 0.00 & 0.00 \\
\hline Pace et al. ${ }^{23} 2008$ & 9 & 11.10 & 0.00 & 0.00 & 11.10 & 0.00 \\
\hline Alijotas et al. ${ }^{24} 2008$ & $9^{* *}$ & 11.10 & 11.10 & 0.00 & 0.00 & 0.00 \\
\hline Paradela et al..25 2008 & 10 & $\mathrm{NR}$ & 100.00 & 0.00 & 0.00 & 0.00 \\
\hline Song et al. ${ }^{26} 2009$ & 102 & 2.94 & 2.94 & 0.00 & 0.00 & 0.00 \\
\hline Cheng et al. ${ }^{27} 2010$ & 195 & 2.05 & 2.05 & 0.00 & 0.00 & 0.00 \\
\hline Sopeña et al. ${ }^{28} 2012$ & 17 & 23.50 & 11.70 & 5.80 & 5.80 & 0.00 \\
\hline Dumas et al. ${ }^{3} 2014$ & 91 & 25.20 & 2.20 & 11.00 & 12.00 & 0.00 \\
\hline Sopeña et al. ${ }^{29} 2017$. & 113 & NR & 31.20 & 51.30 & 17.50 & 1.76 \\
\hline Rue et al. ${ }^{33} 2016$ & 1 & \multicolumn{4}{|c|}{ LES con SAF complicado con EKF } & $\begin{array}{l}\text { Isquemia vascular } \\
\text { retiniana }\end{array}$ \\
\hline Kinouchi et al..$^{34} 2018$ & 1 & \multicolumn{4}{|c|}{ LES complicado con EKF } & $\begin{array}{c}\text { Retinopatía } \\
\text { vaso-oclusiva }\end{array}$ \\
\hline
\end{tabular}

* De 7.3 subió a 9\%, debido a que al momento del diagnóstico cuatro pacientes se consideraron lupus incompleto. * Reporte de casos con EKF durante el embarazo. $\mathrm{NR}=$ no reportada; EKF = enfermedad de Kikuchi-Fujimoto; LES = lupus eritematoso sistémico; SAF = síndrome de anticuerpos antifosfolípidos.

nes basales y rutinarias al establecer el diagnóstico de LES, más aún cuando la presentación es inusual como en la EFK, para evitar daño irreversible. Conocer las características clínicas de esta enfermedad puede ayudar a los profesionales de la salud a llegar al diagnóstico correcto.

Limitaciones: una limitación importante es la falta de realización de inmunohistoquímica en la biopsia de ganglio para determinar expresión de CD68.

Consentimiento informado: los padres de la paciente firmaron la carta de consentimiento informado para la publicación de caso.

Financiación: no se utilizaron fuentes de financiamiento para la realización de este artículo.

Conflicto de intereses: los autores declaran que no existe conflicto de intereses de ningún tipo en el trabajo realizado.

\section{BIBLIOGRAFÍA}

1. Kikuchi M. Lymphadenitis showing focal reticulum cell hyperplasia with nuclear debris and phagocytosis. Nippon Ketsueki Gakkai Zasshi. 1972; 35: 379-380.

2. Fujimoto Y, Kozima Y, Yamaguchi K. Cervical subacute necrotizing lymphadenitis. A new clinicopathological entity. Naika. 1972; 20: 920-927.

3. Dumas G, Prendki V, Haroche J, Amoura Z, Cacoub P, Galicier $L$ et al. Kikuchi-Fujimoto disease: retrospective study 91 cases and review of the literature. Medicine. 2014; 93 (24): 372-382. doi: 10.1097/md.0000000000000 220.

4. Kucukardali Y, Solmazgul E, Kunter E, Oncul O, Yildirim S, Kaplan M. Kikuchi-Fujimoto disease: analysis of 244 cases. Clinical Rheumatology. 2006; 26 (1): 50-54. doi: 10.1007/ s10067-006-0230-5.

5. Gallagher K, Viswanathan A, Okhravi N. Association of systemic lupus erythematosus with uveitis. JAMA Ophthalmology. 2015; 133 (10): 1190. doi: 10.1001/ jamaophthalmol.2015.2249. 
Vol. 29, Núm. 2 • Mayo-Agosto 2020

6. Sarre-Álvarez D, Garza-Zúñiga MJ, Rodríguez-Weber F, Díaz-Greene E. Enfermedad de Kikuchi-Fujimoto. Med Int Méx. 2015; 31: 402-413.

7. Dorfman RF, Berry GJ. Kikuchi's histocytic necrotizing lymphadenitis: an analysis of 108 cases with emphasis on differential diagnosis. Semin Diagn Pathol. 1988; 5 (4): 329-345.

8. Hu S, Kuo T, Hong HS. Lupus lymphadenitis simulating Kikuchi's lymphadenitis in patients with systemic lupus erythematosus: A clinicopathological analysis of six cases and review of the literature. Pathology International. 2003; 53 (4): 221-226. doi: 10.1046/j.1320-5463.2003.01458.x.

9. Pileri S, Kikuchi M, Helbron D, Lennert K. Histiocytic necrotizing lymphadenitis without granulocytic infiltration. Virchows Arch A Pathol Anat Histol. 1982; 395 (3): 257-271.

10. Turner RR, Martin J, Dorfman RF. Necrotizing lymphadenitis. A study of 30 cases. Am J Surg Pathol. 1983; 7: 115-123.

11. Cho KJ, Kim CW, Park SH, Lee SK. Necrotizing lymphadenitis: a clinicopathologic study of 36 cases with immunohistochemical analysis. J Korean Med Sci. 1991; 6: 55-61.

12. el-Ramahi KM, Karrar A, Ali MA. Kikuchi disease and its association with systemic lupus erythematosus. Lupus. 1994; 3 (5): 409-411.

13. Kuo TT. Kikuchi's disease (histiocytic necrotizing lymphadenitis). A clinicopathologic study of 79 cases with an analysis of histologic subtypes, immunohistology, and DNA ploidy. Am J Surg Pathol. 1995; 19 (7): 798-809.

14. Chen YH, Lan JL. Kikuchi disease in systemic lupus erythematosus: clinical features and literature review. J Microbiol Immunol Infect. 1998; 31: 187-192.

15. Lin HC, Su CY, Huang CC, Hwang CF, Chien CY. Kikuchi's disease: a review and analysis of 61 cases. Otolaryngol Head Neck Surg. 2003; 128 (5): 650-653. doi: 10.1016/S01945998(02)23291-X.

16. Santana A, Lessa B, Galrão L, Lima I, Santiago M. Kikuchi-Fujimoto's disease associated with systemic lupus erythematosus: case report and review of the literature. Clin Rheumatol. 2005; 24 (1): 60-63.

17. Lin HC, Su CY, Huang SC. Kikuchi's disease in Asian children. Pediatrics. 2005; 115 (1): e92-e96. doi: 10.1542/ peds.2004-0924.

18. Chen HC, Lai JH, Huang GS, Gao HW, Chen CH, Kuo SY et al. Systemic lupus erythematosus with simultaneous onset of Kikuchi-Fujimoto's disease complicated with antiphospholipid antibody syndrome: a case report and review of the literature. Rheumatol Int. 2005; 25 (4): 303-306.

19. Yu HL, Lee SS, Tsai HC, Huang CK, Chen YS, Lin HH et al. Clinical manifestations of Kikuchi's disease in southern Taiwan. J Microbiol Immunol Infect. 2005; 38 (1): 35-40.

20. Sanpavat A, Wannakrairot $P$, Assanasen T. Necrotizing nongranulomatous lymphadenitis: a clinicopathologic study of 40 Thai patients. Southeast Asian J Trop Med Public Health. 2006; 37 (3): 563-570.

21. Park HS, Sung MJ, Park SE, Lim YT. Kikuchi-Fujimoto disease of 16 children in a single center of Korea. Pediatr Allergy Immunol. 2007; 18 (2): 174-178.

22. Goldblatt F, Andrews J, Russell A, Isenberg D. Association of Kikuchi-Fujimoto's disease with SLE. Rheumatology (Oxford). 2008; 47 (4): 553-554. doi: 10.1093/rheumatology/ken008.
23. Pace-Asciak P, Black MA, Michel RP, Kost K. Case series: raising aware- ness about Kikuchi-Fujimoto disease among otolaryngologists: is it linked to systemic lupus erythematosus? J Otolaryngol Head Neck Surg. 2008; 37 (6): 782-787.

24. Alijotas-Reig J, Casellas-Caro M, Ferrer-Oliveras R, CaberoRoura L, Vilardell-Tarres M. Recurrent Kikuchi-Fujimoto disease during pregnancy: report of case evolving into systemic lupus erythematosus and review of published work. J Obstet Gynaecol Res. 2008; 34 (4 Pt 2): 595-598. doi: 10.1111/j.1447-0756.2008.00727.x.

25. Paradela S, Lorenzo J, Martínez-Gómez W, Yebra-Pimentel $\mathrm{T}$, Valbuena L, Fonseca E. Interface dermatitis in skin lesions of Kikuchi-Fujimoto's disease: a histopathological marker of evolution into systemic lupus erythematosus? Lupus. 2008; 17 (12): 1127-1135. doi: 10.1177/0961203308092161.

26. Song JY, Lee J, Park DW, Sohn JW, Suh SI, Kim IS et al. Clinical outcome and predictive factors of recurrence among patients with Kikuchi's disease. Int J Infect Dis. 2009; 13 (3): 322-326. doi: 10.1016/j.ijid.2008.06.022.

27. Cheng CY, Sheng WH, Lo YC, Chung CS, Chen YC, Chang SC. Clinical presentations, laboratory results and outcomes of patients with Kikuchi's disease: emphasis on the association between recurrent Kikuchi's disease and autoimmune diseases. J Microbiol Immunol Infect. 2010; 43 (5): 366-371. doi: 10.1016/S1684-1182(10)60058-8.

28. Sopeña B, Rivera A, Vázquez-Triñanes C, Fluiters E, González-Carreró J, del Pozo M et al. Autoimmune manifestations of Kikuchi disease. Semin Arthritis Rheum. 2012; 41 (6): 900-906. doi: 10.1016/j.semarthrit.2011.11.001.

29. Sopeña B, Rivera A, Chamorro A, Freire M, Alende V, Seco $E$ et al. Clinical association between Kikuchil disease and systemic lupus erythematosus: A systematic literature review. Semin Arthritis Rheum. 2017; 47 (1): 46-52. doi: 10.1016/j. semarthrit.2017.01.011.

30. Semon HC, Wolff E. Acute lupus erythematosus, with fundus lesions. Proc R Soc Med. 1933; 27: 153-157.

31. Silpa-archa S, Lee JJ, Foster CS. Ocular manifestations in systemic lupus erythematosus. Br J Ophthalmol. 2016; 100 (1): 135-141.

32. Zou W, Wen F. Bilateral occlusive retinal vasculitis in KikuchiFujimoto disease. Clin Exp Ophthalmol. 2007; 35 (9): 875877. doi: 10.1111/j.1442-9071.2007.01637.x.

33. Rue KS, Rodger DC, Rao NA. Retinopathy in lupus transitioned to Kikuchi-Fujimoto disease. Am J Ophthalmol Case Rep. 2016; 3: 43-46. doi: 10.1016/j.ajoc.2016.06.004.

34. Kinouchi R, Kinouchi M, Ishibazawa A, Yoshida A. Macular capillary recovery in systemic lupus erythematosus complicated by Kikuchi-Fujimoto disease. Int Ophthalmol. 2018; 38 (4): 1797-1801. doi: 10.1007/s10792-017-0650-z.

Dirección para correspondencia:

Dr. José Guillermo Murguía Pérez

Hospital Pediátrico de Sinaloa.

Blvd. Constitución s/n, Col. Almada,

Culiacán, Sinaloa, México.

Teléfono: +52 0443122100155

E-mail: murguia_jose@ucol.mx

Orcid: 0000-0002-2587-8405 